\title{
Pilot Designs with Guard Bands for Channel Estimation of MIMO OFDM Systems with Frequency-Dependent I/Q Imbalances
}

\author{
Daniel Munoz and Hlaing Minn \\ Department of Electrical Engineering, University of Texas at Dallas \\ E-mail: \{djm072000, hlaing.minn\}@utdallas.edu
}

\begin{abstract}
All practical OFDM systems insert null guard bands to facilitate easy filter implementation and emission spectrum control. The null guard bands introduce a constraint for optimal pilot designs in MIMO OFDM systems. Semiconductor downscaling and the use of high modulation order have signified the importance of pilot designs for OFDM systems in the presence of I/Q imbalances, but existing designs have not addressed the use of guard bands, the overhead efficiency, the estimation optimality, and general applicability in pilot data multiplexed format and/or MIMO setup. This paper proposes a pilot design for channel estimation of MIMO OFDM systems with guard bands and both transmit and receive frequency-dependent $I / Q$ imbalances, which overcomes the limitations of the existing designs. Simulation and numerical results corroborate better performance, overhead advantage and applicability in pilot-data-multiplexed MIMO setup of the proposed design over the existing MIMO OFDM pilot design with $\mathrm{I} / \mathrm{Q}$ imbalances.

Index Terms - Channel estimation, Guard band, I/Q imbalance, MIMO, OFDM, Pilot design
\end{abstract}

\section{INTRODUCTION}

Channel estimation, crucial for coherent detection, is typically accomplished by the aid of pilot tones in practical OFDM systems. Pilot designs with performance optimality and minimal overhead are much desirable especially for multiinput multi-output (MIMO) or multiple antennas systems since MIMO systems are more sensitive to estimation errors and they have a multitude of channel parameters. There exist several pilot designs for MIMO OFDM systems in the literature (e.g., [1], [2] and references therein). However, these designs do not consider I/Q imbalances (mismatches between the inphase and the quadrature branches of the transceiver). The detrimental effects of I/Q imbalances become more significant due to the semiconductor downscaling and the use of high order modulation [3]. This mandates development of efficient pilot designs for channel estimation of MIMO OFDM systems with I/Q imbalances. All practical OFDM systems exclude some subcarriers at the band edges from use, as seen in [4] where for a FFT size of 128 , only 73 subcarriers are allocated for data and pilot use. This introduces an additional constraint in the pilot design.

Many papers have addressed the I/Q imbalance issue and proposed pilot designs which either estimate the I/Q imbalance parameters and try to remove its effects from the channel (see [5]-[9] , or estimate the combined response of the channel and I/Q imbalance (e.g. [10]-[17]). In this paper, we consider the latter approach. Among the existing designs, only [17] addresses MIMO systems. However, it does not consider guard bands in the design. Although [16] applies a guard band in its simulation, its pilot design does not consider guard bands, and it is only for SISO systems.

In this paper we develop a pilot design for MIMO OFDM systems with guard bands in the presence of transmit and receive frequency-dependent I/Q imbalances. The proposed design is applicable to MIMO OFDM systems with guard bands and both transmit and receive frequency-dependent I/Q imbalances while maintaining estimation performance optimality, minimal overhead, and generality in transmission format (e.g., pilot-data multiplexed setup), hence overcoming the limitations and disadvantages of the existing methods.

The rest of the paper is organized as follows. Section II describes signal model in the presence of frequency-independent and -dependent transmit and receive I/Q imbalances in OFDM systems. Section III presents pilot design criteria. In Section IV, we present our proposed pilot design. Performance evaluation and simulation results are discussed in Section V, and conclusions are provided in Section VI.

\section{SignAl MODEL}

First, consider a single antenna system with both transmit and receive I/Q imbalances where $\left\{a_{t}^{I}, a_{t}^{Q}\right\}$ and $\left\{\theta_{t}^{I}, \theta_{t}^{Q}\right\}$ represent frequency-independent gains and phase offsets of the I and Q branches at the transmitter. The equivalent pulse shaping filters (the overall impulse responses including DAC, amplifier, pulse shaping, and frequency-dependent I/Q imbalances) for the I and Q branches of the transmitter are denoted by $g_{t}^{I}(t)$ and $g_{t}^{Q}(t)$. The receiver parameters are defined in the same manner with the subscript $t$ replaced by $r$. Then the transmit system can be viewed as the summation of two systems namely direct system whose input is the same as the transmitter input signal $s(t)=s_{I}(t)+j s_{Q}(t)$ and mirror system whose input is $s^{*}(t)$. The impulse responses of the direct and mirror systems at the transmitter are denoted by $g_{T}^{D}(t)$ and $g_{T}^{M}(t)$, and those at the receiver are $g_{R}^{D}(t)$ and $g_{R}^{M}(t)$, respectively. They are related to the I/Q imbalance parameters and the equivalent pulse shaping filters as

$$
\begin{aligned}
g_{T}^{D}(t) & =\left[a_{t}^{I} e^{j \theta_{t}^{I}} g_{t}^{I}(t)+a_{t}^{Q} e^{j \theta_{t}^{Q}} g_{t}^{Q}(t)\right] / 2 \\
g_{T}^{M}(t) & =\left[a_{t}^{I} e^{j \theta_{t}^{I}} g_{t}^{I}(t)-a_{t}^{Q} e^{j \theta_{t}^{Q}} g_{t}^{Q}(t)\right] / 2 \\
g_{R}^{D}(t) & =\left[a_{r}^{I} e^{-j \theta_{r}^{I}} g_{r}^{I}(t)+a_{r}^{Q} e^{-j \theta_{r}^{Q}} g_{r}^{Q}(t)\right] / 2 \\
g_{R}^{M}(t) & =\left[a_{r}^{I} e^{j \theta_{r}^{I}} g_{r}^{I}(t)-a_{r}^{Q} e^{j \theta_{r}^{Q}} g_{r}^{Q}(t)\right] / 2 .
\end{aligned}
$$

The channel impulse response $h(t)$ is the low-pass-equivalent of the bandpass channel $h_{\mathrm{bp}}(t)$. 
In MIMO systems, different RF chains corresponding to different antennas may give rise to different I/Q imbalances. The above impulse responses corresponding to the $i$ th transmit antenna and the $j$ th receive antenna are denoted by $g_{T, i}^{D}(t)$, $g_{T, i}^{M}(t), g_{R, j}^{D}(t)$, and $g_{R, j}^{M}(t)$, respectively. A simplified lowpass-equivalent signal model for MIMO is presented in Fig. 1 for the $j$ th receive antenna where the impulse responses of the overall direct channel $p_{i, j}(t)$ and the overall mirror channel $q_{i j}(t)$ corresponding to the $i$ th transmit antenna and the $j$ th receive antenna read as

$$
\begin{aligned}
p_{i j}(t) & =g_{T, i}^{D}(t) * h_{i j}(t) * g_{R, j}^{D}(t) \\
& +\left(g_{T, i}^{M}(t)\right)^{*} * h_{i j}^{*}(t) * g_{R, j}^{M}(t) \\
q_{i j}(t) & =g_{T, i}^{M}(t) * h_{i j}(t) * g_{R, j}^{D}(t) \\
& +\left(g_{T, i}^{D}(t)\right)^{*} * h_{i j}^{*}(t) * g_{R, j}^{M}(t) .
\end{aligned}
$$

The receive filter output signal corresponding to the receive antenna $j$ and transmit signals $\left\{s_{i}(t)\right\}$ is

$$
r_{j}(t)=\sum_{i=0}^{N_{\mathrm{Tx}}-1}\left(s_{i}(t) * p_{i j}(t)+s_{i}^{*}(t) * q_{i j}(t)\right)+n_{j}(t)
$$

where the complex Gaussian noise $n_{j}(t)$ is given by

$$
n_{j}(t)=w(t) * g_{R, j}^{D}(t)+w^{*}(t) * g_{R, j}^{M}(t) .
$$

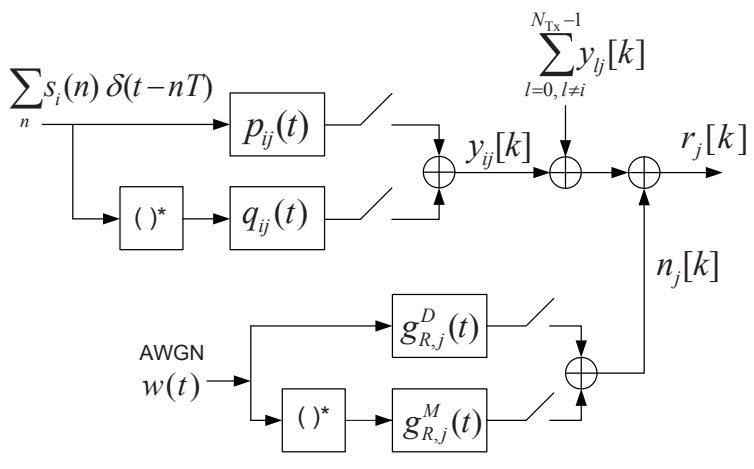

Fig. 1. A simplified equivalent lowpass MIMO system model with frequencyindependent and frequency-dependent I/Q imbalances

When MIMO channels are independent or their joint statistics are unknown, the logical approach to the estimation of the equivalent direct and mirror channels is to estimate them at each receive antenna independently. Hence, we just need to describe them for one receive antenna. In the following we drop the receive antenna index $j$. We consider an OFDM system with a cyclic prefix $(\mathrm{CP})$ interval ( $N_{\mathrm{CP}}$ samples) longer than the maximum span ( $L$ samples) of $\left\{p_{i}(t)\right\}$ and $\left\{q_{i}(t)\right\}$.

Now, let us consider a low-pass-equivalent discrete-time OFDM system with $N$ subcarriers. The channels are assumed to be constant over $K$ symbol intervals. The discrete-time transmit training signal from the $i$ th transmit antenna during the $l$ th symbol duration is denoted by $s_{i}^{(l)}[k]$ with the integer index $k \in\left[-N_{\mathrm{CP}}, N-1\right]$, and the CP samples $s_{i}^{(l)}[m]=$ $s_{i}^{(l)}[N-m]$ for $m \in\left[-N_{\mathrm{CP}},-1\right]$. Similar notations apply to data signal $x_{i}^{(l)}[k]$. The discrete-time versions of $p_{i}(t)$ and $q_{i}(t)$ are denoted by $L \times 1$ vectors $\boldsymbol{p}_{i}$ and $\boldsymbol{q}_{i}$, respectively. The time-domain $N \times 1$ received signal vector after $\mathrm{CP}$ removal at each receive antenna for the $l$ th OFDM symbol is denoted by $\boldsymbol{r}_{l}$ which can be expressed in a general pilot-data multiplexed setup (which includes pilot only or data only symbols as special cases) as

$$
\boldsymbol{r}_{l}=\sum_{i=0}^{N_{\mathrm{Tx}}-1}\left\{\left(\boldsymbol{S}_{i}[l]+\boldsymbol{X}_{i}[l]\right) \boldsymbol{p}_{i}+\left(\boldsymbol{S}_{i}^{*}[l]+\boldsymbol{X}_{i}^{*}[l]\right) \boldsymbol{q}_{i}\right\}+\boldsymbol{n}_{l}
$$

where $(m, k)$ th element of the $N \times L$ signal matrix $\boldsymbol{S}_{i}[l]$ (or $\left.\boldsymbol{X}_{i}[l]\right)$ is $s_{i}^{(l)}[m-k]$ (or $x_{i}^{(l)}[m-k]$ ) with $m \in[0, N-1]$ and $k \in[0, L-1]$. The received signal vector for $K$ OFDM symbols is given by

$$
\boldsymbol{r}=\boldsymbol{S p}+\boldsymbol{S}^{*} \boldsymbol{q}+\boldsymbol{X} \boldsymbol{p}+\boldsymbol{X}^{*} \boldsymbol{q}+\boldsymbol{n}
$$

where $\boldsymbol{r}=\left[\boldsymbol{r}_{0}^{T} \boldsymbol{r}_{1}^{T} \ldots \boldsymbol{r}_{K-1}^{T}\right]^{T}, \boldsymbol{p}=\left[\boldsymbol{p}_{0}^{T} \boldsymbol{p}_{1}^{T} \ldots \boldsymbol{p}_{N_{\mathrm{Tx}-1}}^{T}\right]^{T}, \boldsymbol{q}=$ $\left[\boldsymbol{q}_{0}^{T} \boldsymbol{q}_{1}^{T} \ldots \boldsymbol{q}_{N_{\mathrm{T} \times-1}}^{T}\right]^{T}, \boldsymbol{n}=\left[\boldsymbol{n}_{0}^{T} \boldsymbol{n}_{1}^{T} \ldots \boldsymbol{n}_{K-1}^{T}\right]^{T}$, the $(k, i)$ th submatrice of $\boldsymbol{S}$ and $\boldsymbol{X}$ are respectively given by $\boldsymbol{S}_{i}[k]$ and $\boldsymbol{X}_{i}[k]$, with $k \in[0, K-1]$ and $i \in\left[0, N_{\mathrm{Tx}}-1\right]$. The complex Gaussian noise vectors $\left\{\boldsymbol{n}_{l}\right\}$ are given by

$$
\boldsymbol{n}_{l}=\boldsymbol{G}_{R, D} \boldsymbol{w}_{l}+\boldsymbol{G}_{R, Q} \boldsymbol{w}_{l}^{*}
$$

where $\left\{\boldsymbol{w}_{l}\right\}$ are independent and identically distributed (iid) random vectors, each consisting of iid circularly-symmetric complex Gaussian random variables with the variance $\sigma_{w}^{2}$. Let $\lambda$ denote the maximum of the number of taps of $g_{R}^{D}[k]$ and $g_{R}^{Q}[k]$. Then, $\boldsymbol{G}_{R, D}$ and $\boldsymbol{G}_{R, Q}$ are $N \times(N+\lambda)$ matrices with their first rows given by $\left[g_{R}^{D}[0], g_{R}^{D}[1], \ldots, g_{R}^{D}[\lambda], \mathbf{0}_{N-1}\right]$ and $\left[g_{R}^{Q}[0], g_{R}^{Q}[1], \ldots, g_{R}^{Q}[\lambda], \mathbf{0}_{N-1}\right]$, respectively, and their next $k$ th rows are cyclically $k$-right-shift versions of their corresponding first rows.

\section{MIMO OFDM PILOT DESIGN CRITERIA}

For coherent detection, the direct channel $\boldsymbol{p}$ and the mirror channel $\boldsymbol{q}$ need to be estimated at the receiver. In practical systems, the statistics of the channel and the transceiver imperfections are unknown and they can be non-stationary as well. This leads to the practical choice of least-squares type channel estimators as considered in this paper. The estimates of the direct and mirror CIR vectors are given by

$$
\begin{aligned}
& \hat{\boldsymbol{p}}=\left(\boldsymbol{S}^{H} \boldsymbol{S}\right)^{-1} \boldsymbol{S}^{H} \boldsymbol{r} \\
& \hat{\boldsymbol{q}}=\left(\boldsymbol{S}^{T} \boldsymbol{S}^{*}\right)^{-1} \boldsymbol{S}^{T} \boldsymbol{r} .
\end{aligned}
$$

Our pilot designs will be based on minimizing the mean-square error (MSE) of the channel estimation.

We observe the following conditions for the pilot designs:

1) Estimation Identifiability Condition: The identifiability of $\boldsymbol{p}$ and $\boldsymbol{q}$ estimation requires that $\boldsymbol{S}^{H} \boldsymbol{S}$ is of full-rank.

2) Zero Cross Channel Interference Condition: The interference between the direct chanel and the mirror channel is completely removed when $\boldsymbol{S}^{H} \boldsymbol{S}^{*}=\mathbf{0}_{L N_{\mathrm{Tx}}}$. 
3) Zero Data Interference Condition: The random data interference is completely suppressed when $\boldsymbol{S}^{H} \boldsymbol{X}=$ $\mathbf{0}_{L N_{\mathrm{Tx}}}$ and $\boldsymbol{S}^{H} \boldsymbol{X}^{*}=\mathbf{0}_{L N_{\mathrm{Tx}}}$.

4) White Noise Optimality Condition: For the scenario where the equivalent receive-filter is a square-root Nyquist filter, the MSE due to the noise is minimized when $\boldsymbol{S}^{H} \boldsymbol{S}=E_{K} \boldsymbol{I}_{L N_{\mathrm{Tx}}}$ where $E_{K}$ is the total energy of the transmit signal vector from a transmit antenna over $K$ symbols (excluding CPs). The frequencyindependent receiver I/Q imbalance with a square-root raised cosine receive filter represents this scenario.

5) For the scenario with frequency-dependent receiver $I / Q$ imbalance, the noise covariance matrix is unknown a priori, and hence it is infeasible to develop optimal pilot designs. However, frequency selectivity of the receiver I/Q imbalance is typically very small. A practical approach in this case is to assume frequency-flat receiver I/Q imbalance in the pilot designs which leads to the requirement of $\boldsymbol{S}^{H} \boldsymbol{S}=E_{K} \boldsymbol{I}_{L N_{\mathrm{Tx}}}$.

When the identifiability and zero data interference condition are satisfied, the MSEs of $\hat{\boldsymbol{p}}$ and $\hat{\boldsymbol{q}}$ becomes

$$
\begin{aligned}
\operatorname{MSE}_{\boldsymbol{p}}= & \operatorname{Tr}\left[\left(\boldsymbol{S}^{H} \boldsymbol{S}\right)^{-1} \boldsymbol{S}^{H} \boldsymbol{S}^{*} E\left[\boldsymbol{q} \boldsymbol{q}^{H}\right] \boldsymbol{S}^{T} \boldsymbol{S}\left(\boldsymbol{S}^{H} \boldsymbol{S}\right)^{-1}\right. \\
+ & \left.\left(\boldsymbol{S}^{H} \boldsymbol{S}\right)^{-1} \boldsymbol{S}^{H} \boldsymbol{C}_{\boldsymbol{n}} \boldsymbol{S}\left(\boldsymbol{S}^{H} \boldsymbol{S}\right)^{-1}\right] \\
\mathrm{MSE}_{\boldsymbol{q}}=\operatorname{Tr} & {\left[\left(\boldsymbol{S}^{T} \boldsymbol{S}^{*}\right)^{-1} \boldsymbol{S}^{T} \boldsymbol{S} E\left[\boldsymbol{p} \boldsymbol{p}^{H}\right] \boldsymbol{S}^{H} \boldsymbol{S}^{*}\left(\boldsymbol{S}^{T} \boldsymbol{S}^{*}\right)^{-1}\right.} \\
+ & \left.\left(\boldsymbol{S}^{T} \boldsymbol{S}^{*}\right)^{-1} \boldsymbol{S}^{T} \boldsymbol{C}_{\boldsymbol{n}} \boldsymbol{S}^{*}\left(\boldsymbol{S}^{T} \boldsymbol{S}^{*}\right)^{-1}\right]
\end{aligned}
$$

where the noise covariance matrix $C_{n}$ is given by

$$
\boldsymbol{C}_{\boldsymbol{n}}=\sigma_{w}^{2}\left(\boldsymbol{G}_{R, D} \boldsymbol{G}_{R, D}^{H}+\boldsymbol{G}_{R, Q} \boldsymbol{G}_{R, Q}^{H}\right) \otimes \boldsymbol{I}_{K} .
$$

If the zero cross channel interference condition is also met, the above MSE expressions become

$$
\begin{aligned}
& \operatorname{MSE}_{\boldsymbol{p}}=\operatorname{Tr}\left[\left(\boldsymbol{S}^{H} \boldsymbol{S}\right)^{-1} \boldsymbol{S}^{H} \boldsymbol{C}_{\boldsymbol{n}} \boldsymbol{S}\left(\boldsymbol{S}^{H} \boldsymbol{S}\right)^{-1}\right] \\
& \mathrm{MSE}_{\boldsymbol{q}}=\operatorname{Tr}\left[\left(\boldsymbol{S}^{T} \boldsymbol{S}^{*}\right)^{-1} \boldsymbol{S}^{T} \boldsymbol{C}_{\boldsymbol{n}} \boldsymbol{S}^{*}\left(\boldsymbol{S}^{T} \boldsymbol{S}^{*}\right)^{-1}\right] .
\end{aligned}
$$

Additionally, if the demodulator output noise samples are white (i.e., $\left.\boldsymbol{C}_{\boldsymbol{n}}=\sigma_{n}^{2} \boldsymbol{I}\right)$, the MSE expressions simplify to $\mathrm{MSE}_{\boldsymbol{p}}=\mathrm{MSE}_{\boldsymbol{q}}=\sigma_{n}^{2} \operatorname{Tr}\left[\left(\boldsymbol{S}^{H} \boldsymbol{S}\right)^{-1}\right]$.

The MIMO OFDM pilot design criterion satisfying estimation identifiability, zero data interference condition, zero cross channel interference, and white noise optimality reads as

$$
\left.\begin{array}{l}
\left(\boldsymbol{S}^{H} \boldsymbol{S}\right)=E_{K} \boldsymbol{I}_{N_{\mathrm{Tx}} L} \quad \& \quad\left(\boldsymbol{S}^{H} \boldsymbol{S}^{*}\right)=\mathbf{0}_{N_{\mathrm{Tx}} L} \\
\boldsymbol{S}^{H} \boldsymbol{X}=\mathbf{0}_{N_{\mathrm{Tx}} L} \quad \& \quad \boldsymbol{S}^{H} \boldsymbol{X}^{*}=\mathbf{0}_{N_{\mathrm{Tx}} L}
\end{array}\right\}
$$

with the constraint that the pilot tones do not occupy the guard bands. The corresponding MSE becomes

$$
\begin{aligned}
\operatorname{MSE}_{\boldsymbol{p}}=\mathrm{MSE}_{\boldsymbol{q}}= & \frac{\sigma_{w}^{2}}{E_{K}^{2}} \operatorname{Tr}\left[\left(\boldsymbol { S } ^ { H } \left(\left(\boldsymbol{G}_{R, D} \boldsymbol{G}_{R, D}^{H}\right.\right.\right.\right. \\
& \left.\left.\left.\left.+\boldsymbol{G}_{R, Q} \boldsymbol{G}_{R, Q}^{H}\right) \otimes \boldsymbol{I}_{K}\right) \boldsymbol{S}\right)^{-1}\right] .
\end{aligned}
$$

\section{Pilot Designs}

For a typical FFT size $N$ (a power of 2), define $L_{0}=$ $2^{\left\lceil\log _{2}(\mathrm{~L})\right\rceil}, L_{i}=2^{i} L_{0}$, and $M_{i}=N / L_{i}$. The pilot tone index set for antenna index $i$ and OFDM symbol index $l$ is represented by $\mathcal{J}_{i, l}$ which consists of non-zero pilot tone index set $\mathcal{J}_{i, l}^{\text {pilot }}$ and the null pilot tone index set $\mathcal{J}_{i, l}^{\text {null }}$. The union of $\mathcal{J}_{i, l}$ across all transmit antennas is denoted by $\mathcal{J}_{l}$. The tone index of the null guard band is defined by $\mathcal{J}^{\text {guard }}$ (if the DC subcarrier is not used, we include it in this set), and the union of data tone index sets from all transmit antennas for OFDM symbol index $l$ is denoted by $\mathcal{I}_{l}$. Let $c_{i, l}[k]$ denote the pilot symbol on the $k$ th subcarrier of the $l$ th OFDM symbol from the $i$ th transmit antenna.

\section{A. Reference Design}

For a comparison, the design in [17] will be used as the reference. This pilot design uses a Hadamard sequence structure for channel estimation in the presence of I/Q imbalance to avoid both inter-carrier and inter-antenna interferences. In our notation, the index sets are given by

$$
\begin{aligned}
\mathcal{J}_{i, l}^{\text {pilot }}= & \{1,2, \ldots N-1\} \backslash \mathcal{J}^{\text {guard }} \text { for } i=0,1, \ldots N_{\mathrm{Tx}} \\
& \text { and } l=0,1 \ldots 2 N_{\mathrm{Tx}}-1 \\
\mathcal{I}_{i, l}= & \emptyset \text { for } i=0,1, \ldots N_{\mathrm{Tx}}-1 \\
& \text { and } l=0,1 \ldots 2 N_{\mathrm{Tx}}-1 \\
\mathcal{J}_{i, l}^{\text {pilot }}= & \emptyset \text { for } i=0,1, \ldots N_{\mathrm{Tx}}-1 \text { and } l \geq 2 N_{\mathrm{Tx}} \\
\mathcal{I}_{i, l}= & \{1,2, \ldots N-1\} \backslash \mathcal{J}^{\text {guard }} \\
& \text { for } i=0,1, \ldots N_{\mathrm{Tx}}-1 \text { and } l \geq 2 N_{\mathrm{Tx}}
\end{aligned}
$$

Define $\boldsymbol{H}$ to be the Hadamard matrix $\left[\begin{array}{cc}1 & 1 \\ 1 & -1\end{array}\right]$. Define $\boldsymbol{C}_{i}$ to be the set of pilots required for $i+1$ antennas. The pilots are given by

$$
\begin{aligned}
c_{0,0}[k] & \in\{-1,1\} \text { for } k \in \mathcal{J}_{0,0}^{\text {pilot }} \\
c_{0,1}[k] & =c_{0,0}[k] \text { for } k \in \mathcal{J}_{0,1}^{\text {pilot }} \text { and } k<N / 2 \\
c_{0,1}[k] & =-c_{0,0}[k] \text { for } k \in \mathcal{J}_{0,1}^{\text {pilot }} \text { and } k>N / 2 \\
\boldsymbol{C}_{i} & =\boldsymbol{H} \otimes \boldsymbol{C}_{i-1} .
\end{aligned}
$$

For example, $\boldsymbol{C}_{0}=\left[\begin{array}{ll}\boldsymbol{c}_{0,0} & \boldsymbol{c}_{0,1}\end{array}\right]$ where $\boldsymbol{c}_{i, l}$ is a row vector comprising of $\left\{c_{i, l}[k], \forall k\right\}$. When $N_{\mathrm{Tx}}=2$, then $\boldsymbol{C}_{1}=$ $\left[\begin{array}{cccc}\boldsymbol{c}_{0,0} & \boldsymbol{c}_{0,1} & \boldsymbol{c}_{0,0} & \boldsymbol{c}_{0,1} \\ \boldsymbol{c}_{0,0} & \boldsymbol{c}_{0,1} & -\boldsymbol{c}_{0,0} & -\boldsymbol{c}_{0,1}\end{array}\right]$ where the columns represent pilots for seperate symbols and the rows represent the pilots for seperate antennas.

In this reference design, excluding subcarriers with index 0 and $N / 2$ violates the pilot design conditions from Section III. In addition, the use of null guard bands causes more deviation from our pilot design conditions, yielding further performance degradation for the reference design. 


\section{B. Proposed Design}

In the proposed design, pilots of different antennas are decoupled by means of time division multiplexing (TDM) while mirror tone interferences are suppressed by means of null tones. All antennas transmit on the same set of subcarriers, but each antenna transmits during a different OFDM symbol. Thus, $K=N_{\text {Tx }}$ OFDM symbols are required to meet the design requirements, while data can be transmitted on other subcarriers in a pilot-data-multiplexed format. Define $\mathcal{T}_{n, k} \triangleq$ $\left[k, k+M_{n}, k+2 M_{n}, \ldots, k+N-M_{n}\right]$ which consists of cyclically equi-spaced $L_{n}$ indexes from $[0, N-1]$. First, obtain $\mathcal{T}_{n, \tau}$ such that

$$
\begin{aligned}
& \left\{\mathcal{T}_{n, \tau} \cap \mathcal{J}^{\text {guard }}\right\}=\emptyset \\
& \tau \in\left\{\left\{1,2, \ldots, M_{n}-1\right\} \backslash\left\{M_{n} / 2\right\}\right\}, \\
& \tau \neq M_{n}-\tau .
\end{aligned}
$$

Define $\quad \tilde{\mathcal{T}}_{n, \tau} \triangleq\left\{N-\mathcal{T}_{n, \tau}\right\}$ modulo $N$.

Then the index sets are given by

$$
\begin{aligned}
& \mathcal{J}_{i, l}^{\text {pilot }}= \begin{cases}\mathcal{T}_{n, \tau}, & \text { if } i=l \\
\emptyset, & \text { else }\end{cases} \\
& \mathcal{J}_{i, l}^{\text {null }}= \begin{cases}\tilde{\mathcal{T}}_{n, \tau}, & \text { if } i=l \\
\mathcal{T}_{n, \tau} \cup \tilde{\mathcal{T}}_{n, \tau}, & \text { else }\end{cases} \\
& \mathcal{I}_{l}=\{0,1,2, \ldots, N-1\} \backslash\left\{\mathcal{I}_{n, \tau} \cup \tilde{\mathcal{T}}_{n, \tau} \cup \mathcal{J}^{\text {guard }}\right\} .
\end{aligned}
$$

The non-zero pilot tones can take on arbitrary phases as

$$
\begin{aligned}
\boldsymbol{c}_{i, i}[k]= & e^{j \phi_{i, k}}, \text { arbitrary } \phi_{i, k}, \\
& k \in \mathcal{J}_{i, i}^{\text {pilot }}, i \in\left\{0,1, \ldots, N_{\mathrm{Tx}}-1\right\} .
\end{aligned}
$$

The assignment of the pilot, null and data indices in (32), (33), and (34) guarentee that the zero data interference condition is met. Due to TDM, there is no mirror tone interference across different antennas (i.e., mirror tone interference suppression through TD) so the estimation identifiability and the white noise optimality conditions are met. The null mirror pilot tones ensure the zero cross channel interference condition is met. To minimize overhead, $M_{n}$ should be set to $M_{0}$. With the DC subcarrier typically set to null, the number of band-edge null guard tones that can be supported by the proposed design without any performance loss is $M_{0}-3$.

\section{Simulation Results And Discussions}

System parameters in the simulation are $N=128,13$ null guard tones at the band edges, 16-QAM, and a Rayleigh fading channel with 4 uncorrelated channel taps having an exponential power delay profile ( $3 \mathrm{~dB}$ per tap decay factor). The DC subcarrier is not used as typical in practical OFDM systems. The frequency-independent I/Q imbalances are set to $\alpha=\frac{a_{t}^{I}}{a_{t}^{Q}}=\frac{a_{r}^{I}}{a_{r}^{Q}}=1.0965(0.4 \mathrm{~dB})$, and $\Delta \theta=\theta_{t}^{I}-\theta_{t}^{Q}$ $=\theta_{r}^{I}-\theta_{r}^{Q}=3^{\circ}$. The frequency-dependent I/Q imbalances are modelled by 3-tap filters with discrete-time impulse responses of $[0.01,0.9999,0.01]$ and $[0.015,0.9998,0.01]$ for the transmit I and Q branches, and [0.012, 0.9997, 0.018], and $[0.01,0.9997,0.02]$ for the receive I and Q branches. As a
TABLE I

Pilot Overhead COMPARISON FOR A MIMO SySTEM WITH $N_{\text {Tx }}$ TRANSMIT ANTENNAS

\begin{tabular}{||c|c|}
\hline Design & Overhead (\# of Tones) \\
\hline Proposed & $2 N_{\mathrm{Tx}} L_{0}$ \\
\hline Reference [17] & $2 N_{\mathrm{Tx}} N_{\text {used }}$ \\
\hline
\end{tabular}

reference, we evaluate the design proposed in [17] with null guard tones according to the above setting. In all methods, the maximum likelihood detection is applied. In the reference method, the energy of each non-zero pilot tone is kept the same as that of data tone. For a fair comparison, total pilot energies of the two methods are set the same. The MIMO system consists of two transmit antennas and two receive antannas.

Fig. 2 shows the total channel estimation MSE $\left(\mathrm{MSE}_{p}+\right.$ $\operatorname{MSE}_{q}$ ) for the proposed design and the reference as well as the theoretical MSEs from Eqns. (14), (15), and (20). The theoretical and simulation MSEs are essentially the same. Due to the null guard tones, the reference design loses estimation optimality slightly. In Fig. 3, the theoretical channel MSE is plotted against the number of guard tones. Since the reference design depends on all subcarriers being utilized, the performance degrades from the optimum MSE performance achieved by the propsed design as the number of guard tones increases. Finally, in Fig. 4, the (uncoded) BER performances of the two pilot designs are compared. The proposed design slightly outperforms the reference design due to the better estimation performance.

Table I compares pilot overhead of the proposed designs and the reference design. The proposed designs require only $2 N_{\mathrm{Tx}} L_{0}$ tones while the reference design uses $2 N_{\mathrm{Tx}} N_{\text {used }}$ tones where $N_{\text {used }}$ is the total number of subcarriers excluding null guard tones and the DC tone. Since $L_{0}$ is typically significantly less than $N_{\text {used }}$ (with our simulation parameters, $L_{0}=8$ and $N_{\text {used }}=114$ ), this demonstrates a substantial reduction in overhead for the proposed design, while providing better BER performance and applicability in pilot-data-multiplexed format.

\section{CONClusions}

The presence of more guard tones causes larger performance degradation to the existing reference pilot design for MIMO OFDM systems with I/Q imbalance, but for typical system settings, its performance loss is small. Hence, its main limitations lie with large pilot overhead and inapplicability to pilot-datamultiplexed format. Formulating in a pilot-data-multiplexed signal model, we have presented a new pilot design for estimating the channel in MIMO OFDM systems affected by transmit and receive frequency-dependent I/Q imbalances in a multipath fading channel. This pilot design is applicable to pilot-data multiplexed systems and provides minimal overhead without loss of optimal channel estimation for systems with up to $\left(M_{0}-3\right)$ band-edge null guard tones. Other pilot designs (e.g. coding across time) are possible to achieve our design criteria with null guard tones and more details are under development. 


\section{REFERENCES}

[1] H. Minn and N. Al-Dhahir, "Optimal training signals for MIMO OFDM channel estimation," IEEE Trans. Wireless Commun., vol. 5, no. 5, pp. 1158-1168, May 2006.

[2] I. Barhumi, G. Leus, and M. Moonen, "Optimal training design for MIMO OFDM systems in mobile wireless channels," IEEE Trans. Signal Process., vol. 51, no. 6, pp. 1615-1624, Jun. 2003.

[3] B. Razavi, "RF Microelectronics," Prentice-Hall, NJ, 1998.

[4] The Mobile Broadband Evolution: 3GPP Release 8 and Beyond: HSPA+, SAE/LTE and LTE-Advanced, 3G Americas, 2009

[5] C. L. Liu, "Impacts of I/Q imbalance on QPSK-OFDM-QAM detection," IEEE Trans. Consum. Electron., vol. 44, no. 3, pp. 984-989, Aug. 1998.

[6] M. Valkama, M. Renfors, and V. Koivunen, "Compensation of frequency-selective I/Q imbalances in wideband receivers: models and algorithms," IEEE SPAWC, Mar. 2001, pp. 42-45.

[7] J. Tubbax, B. Come, L. Van der Perre, S. Donnay, M. Engels, M. Moonen, and H. De Man, "Joint compensation of IQ imbalance and frequency offset in OFDM systems," RAWCON, 2003, pp. 39-42.

[8] A. Tarighat and A. H. Sayed, "Joint compensation of transmitter and receiver impairments in OFDM systems," IEEE Trans. Wireless Commun., vol. 6, no. 1, pp. 240-247, Jan. 2007.

[9] D. Tandur and M. Moonen, "Joint adaptive compensation of transmitter and receiver IQ imbalance under carrier frequency offset in OFDMbased systems," IEEE Trans. Signal Process., 2007,

[10] W. Kirkland and K. Teo, "I/Q distortion correction for OFDM direct conversion receiver," Electronics Letters, 2003, vol. 39, pp. 131-133.

[11] L. Brotje, S. Vogeler, K-D. Kammeyer, "Estimation and correction of transmitter-caused I/Q imbalance in OFDM systems, Proc. 7th Intl. OFDM Workshop, Sept. 2002, pp. 178-182.

[12] Y. Egashira, Y. Tanabe, and K. Sato, "A novel IQ imbalance compensation method with pilot-signals for OFDM system," IEEE VTC Fall, 2006, pp. $1-5$.

[13] A. Tarighat, R. Bagheri, and A. Sayed, "Compensation schemes and performance analysis of IQ imbalances in OFDM receivers," IEEE Trans. Signal Process., 2005, vol. 53, pp. 3257-3268.

[14] R. Chrabieh and S. Soliman, "IQ imbalance mitigation via unbiased training sequences," Proc. IEEE GLOBECOM, 2007, pp. 4280-4285.

[15] E. Lopez-Estraviz, S. De Rore, F. Horlin, and L. Van der Perre, "Optimal training sequences for joint channel and frequency-dependent IQ imbalance estimation in OFDM-based receivers," IEEE ICC 2006, vol. 10 , pp. $4595-4600$.

[16] E. Lopez-Estraviz, S. De Rore, F. Horlin, and A. Bourdoux, "Pilot design for joint channel and frequency-dependent transmit/receive IQ imbalance estimation and compensation in OFDM-based transceivers," IEEE ICC 2007, pp. 4861-4866.

[17] T. Schenk, P. Smulders, and E. Fledderus, "Estimation and compensation of frequency selective TX/RX IQ imbalance in MIMO OFDM systems," IEEE ICC 2006, vol. 1, pp. 251-256.

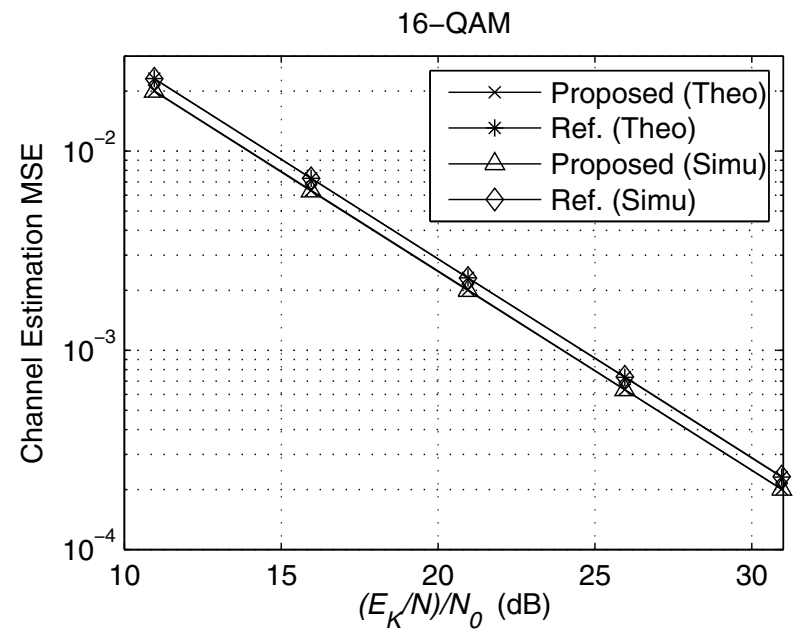

Fig. 2. Estimation MSE comparison for different pilot designs in a $2 \times 2$ MIMO OFDM system

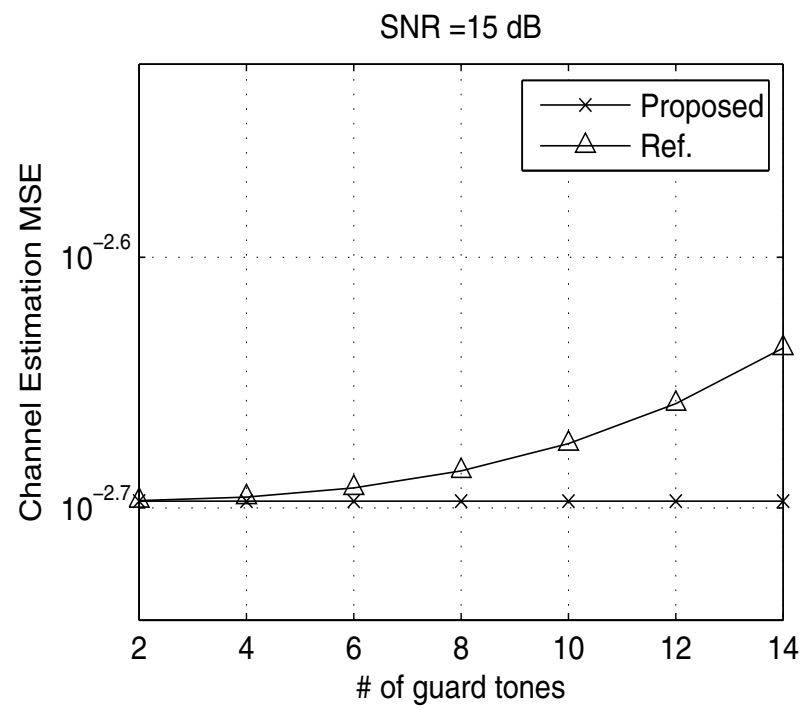

Fig. 3. Estimation MSE comparison varying number of guard tones in a $2 \times 2$ MIMO OFDM system

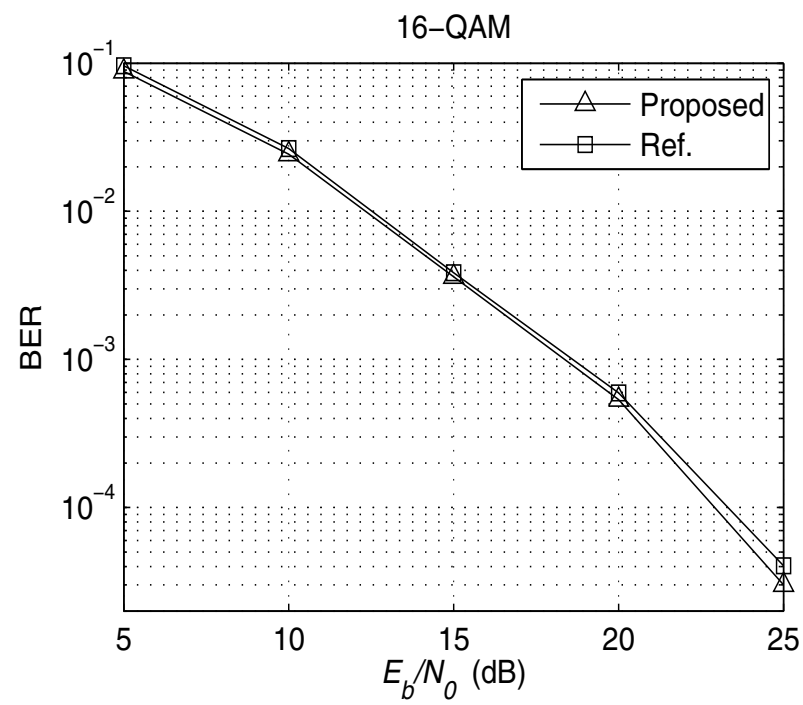

Fig. 4. BER comparison of different pilot designs for a $2 \times 2$ MIMO OFDM system with 16-QAM 